Reviews and Meta-Analysis

\title{
Twenty years of universal vaccination against hepatitis B in Italy: achievements and challenges
}

\author{
Luisa Romanò, Sara Paladini, Alessandro R. Zanetti \\ Dipartimento di Sanità Pubblica - Microbiologia - Virologia, Università di Milano, Italy
}

\begin{abstract}
Significance for public health
This study is a historical overview on the global impact of vaccination against hepatitis B. Viral hepatitis B is a leading cause of acute and chronic liver disease, including cirrhosis and liver cancer. Hepatitis B is the most effective measure to control and prevent hepatitis B and its long-term sequelae. According to the WHO, 179 countries have currently implemented universal vaccination programmes worldwide, and Italy was one of the first countries to introduce a universal strategy of hepatitis B vaccination. On the whole, a significant reduction in newly acquired hepatitis $\mathrm{B}$, carrier rate, and hepatitis B-related mortality has been observed in countries where vaccination has been implemented. In Italy, universal vaccination started in infants as well as adolescents in 1991 with an outstanding record of safety and efficacy. Data collected from Italy and elsewhere clearly show the success of this vaccination in terms of public health since its implementation has dramatically contributed to the saving of lives, prevention of suffering, and savings to the community at large.
\end{abstract}

\begin{abstract}
Viral hepatitis B is a vaccine-preventable disease. Vaccination has proved to be safe and highly effective in reducing the incidence, the carrier rate and HBV-related mortality on a global scale. In Italy, universal vaccination against hepatitis B started in 1991 in infants as well as in adolescents, providing an outstanding record of safety and effectiveness. Within a few years, over $95 \%$ coverage was consistently reported. Today, some 17 million people are immune against hepatitis $\mathrm{B}$ and their immunity has been shown to be long-lasting. At present, no booster is required in healthy vaccinated people to sustain protection. Surveillance data from Italy have shown a clear overall decline in hepatitis B among successfully vaccinated individuals. Furthermore, a generation of children and young people (at present cohorts ranging from 0 to 32 years) is emerging with practically no markers of HBV infection. Italy's vaccination programme has resulted in substantial progress towards the prevention and control of hepatitis $\mathrm{B}$. The vaccination programme must continue. Maintaining mandatory vaccination of infants and increasing $\mathrm{HBV}$ vaccination coverage in high-risk groups, including households of HBsAg carriers as well as immigrants, remain a priority for the future.
\end{abstract}

\section{Introduction}

Viral hepatitis type B is still a major public health problem on a global scale. Infection with hepatitis B virus (HBV) can progress to chronic liver disease, including cirrhosis and hepatocellular carcinoma (HCC), which ranks as the third cause of cancer deaths worldwide.

Vaccination is the most effective measure to control and prevent hepatitis B in terms of both cost- effectiveness and benefit-cost ratios. Safe and effective vaccines have been available since the early 1980 s when the so-called plasma-derived vaccines were first introduced and then largely replaced by yeast-derived recombinant DNA vaccines in the mid-1980s.

Strategies of vaccination were initially targeted toward groups at increased behavioural (e.g. injecting drug users, sexually active homosexual men, people with multiple sexual partners), professional (e.g. health-care workers) risk of infection, to patients like haemophiliacs and those on dialysis maintenance, and to babies born to hepatitis B surface antigen (HBsAg) positive mothers. ${ }^{1}$ Failure of such policies of vaccination to reduce the impact of hepatitis $B$ in the population at large led to the WHO recommendation that all countries introduce a policy of universal infant or adolescent (or both if economically feasible) hepatitis B vaccination integrated into their national immunisation programme. At present, 179 countries worldwide have so far implemented this recommendation or are planning to do. ${ }^{2,3}$ In the WHO European region, UK and Scandinavian countries still do not advocate universal vaccination and have opted (on economic grounds) for targeted vaccination focussed on well-defined risk groups.

Globally, several hundred million vaccinations have been administered worldwide so far, with an outstanding record of safety and efficacy. Vaccination against hepatitis B is well tolerated. Local side effects are generally mild and confined to symptoms at the site of injection, while systemic reactions are uncommon. A number of case reports from France in 1998 and thereafter raised concern that hepatitis B vaccination may lead to new cases or relapses of multiple sclerosis or other neurological disorders, but no clear causal link was established. ${ }^{4-9}$

There is evidence to indicate that anti-HBs (hepatitis B surface antibodies) seroprotection (concentration $\geq 10 \mathrm{mU} / \mathrm{mL}$ ) rates are close to $100 \%$ in healthy children and over $95 \%$ in healthy adults, and that protective antibodies are long-lasting since they may persist for at least $15-20$ years. ${ }^{10-15}$ Vaccinees who have lost antibody usually show a rapid anamnestic response when boosted. ${ }^{13,14,16-18}$ This means that the immunological memory outlasts antibody detection providing protection against clinical disease and the development of carrier state. ${ }^{19-21}$ Thus, current evidence shows that there is no need for a booster in the general population. However, some long-term protection studies have recently shown an increasing number of people with no evidence of boostability (failure to respond to a booster); the reason for this finding and its significance should be further investigated. ${ }^{13,16,22,23}$

Vaccination has been proved to reduce the incidence, carrier rates, and mortality related to hepatitis B. ${ }^{24}$ In Taiwan, where mass vaccination of newborns was first introduced in 1984, the HBsAg carrier rates among children dropped from $9.8 \%$ in the year prior immunisation to $0.6 \%$ in 2004. ${ }^{25}$ Furthermore, the annual average incidence of HCC declined significantly after the implementation of vaccination, demonstrating that hepatitis B vaccine can prevent a major human cancer. $^{26,27}$ Likewise, in other hyperendemic countries such as the Gambia, Malaysia and Alaska, vaccination has clearly proven to be very 
successful. ${ }^{28-30}$ On the whole, following vaccination, remarkable progress has been achieved in terms of global control and prevention of hepatitis B. However, much remains to be done to meet the WHO goal by overriding the economic barriers that still hamper the introduction of this vaccination in some countries with low resources and high endemicity.

\section{The Italian vaccination programme and its impact}

A vaccine against hepatitis B was first introduced in Italy in 1981 for the immunisation of haemodialysis patients and their staff. ${ }^{31}$ In 1983 , a selective programme started using the vaccine in targeted campaigns among at-risk populations. The results encouraged a broader introduction of the vaccine and in 1988 a decree identified subjects at increased risk for hepatitis B to whom vaccination would be offered free of charge. Then, in 1991, a law was passed mandating the universal vaccination of infants and 12-year old adolescents (restricted to the first 12 years of the implementation of the vaccination law) on a national scale. ${ }^{32-35}$ This programme also includes mandatory screening for HBsAg of pregnant women in order to identify babies in need of treatment with hepatitis B immune globulins (HBIG) and vaccine at birth, and recommendation for vaccination of high-risk groups free of charge. By the end of 2003, the first infant cohort vaccinated in 1991 reached the age (12 years) at which adolescent vaccination was programmed. Thus, in 2004, vaccination of 12-year old adolescents was stopped and that of infants maintained.

Before the mandatory vaccination, a wealth of published epidemiological and clinical data on the national burden of the disease and those collected by the National Surveillance System for Acute Viral Hepatitis (SEIEVA) prepared the ground by studiously tracking the presence of hepatitis B in the country, emphasizing the need to urgently implement control and preventive measures.

Take-up of the vaccine was rapid and within a few years over $95 \%$ coverage was consistently reported. Today, some 17 million young people are immune against hepatitis $B$ and their immunity has been shown to be long-lasting. ${ }^{13}$ Data collected prior- and post-vaccination by SEIEVA, clearly show that rates of hepatitis B virus infection fell dramatically. Although the decline in incidence of hepatitis B had started before the mandatory introduction of the vaccine as a result of improving socioeconomic conditions, demographic changes (smaller families), better hygiene and the changes in risky behaviour resulting from anti-AIDS campaigns, refinement in blood screening, and adoption of universal precautions in medical settings, the vaccine ensured that the efforts made towards elimination of the disease were worthwhile. Since the mandatory introduction of universal hepatitis B vaccination, the incidence of the disease has fallen from 5.1 per 100,000 inhabitants in 1991 to 0.9 cases per 100,000 in 2010 . The decline was even more remarkable in 15-24-year-old individuals where incidence per 100,000 dropped from 17 to less than 0.5 in the same period of time. ${ }^{36}$ Furthermore, prevalence of markers of $\mathrm{HBV}$ infection (HBsAg and/or anti-HBc) declined dramatically after the introduction of the vaccination programme. ${ }^{37}$ In addition to the decline of hepatitis $B$, cases of hepatitis delta, which depends on the presence of the hepatitis B virus, are now rarely reported. ${ }^{38}$

Cases of hepatitis B in vaccinated individuals have rarely been reported to SEIEVA and they were generally confined to those who did not complete the vaccination schedule properly. Infections caused by HBVmutants, including the prototype glycine to arginine substitution G145R (first identified in Italy some 25 years ago in babies born to HBsAg car- rier mothers and treated at birth with HBIG and vaccine), are very occasionally the causes of breakthrough infections in successfully vaccinated people. $^{39-42}$ Despite the initial concern that these S-gene mutants could evade vaccine-induced immunity and infect vaccinated people, for the moment, they are not known to pose a public health threat.

\section{The issue of hexavalent vaccines}

In 2000, two hexavalent vaccines (Hexavac, Sanofi Pasteur MSD and Infanrix, Glaxo Smith Kline) were licensed in Europe for vaccinating children against diphtheria, pertussis, poliomyelitis, hepatitis B, and invasive infections caused by Haemophilus influenzae b. In 2005, Hexavac was suspended by the European Medicines Agency (EMA) due to concerns about long-term protection against hepatitis B. ${ }^{43}$ No action was taken over Infanrix since immunogenicity of its hepatitis B component did not raise equal concern. Until suspension, approximately 10 million doses of Hexavac had been distributed globally, of which almost $90 \%$ were used for immunisation of infants in Germany, Austria, and Italy. So the crucial question was whether infants vaccinated with Hexavac were protected or required a booster vaccination to sustain immunity. A large randomised, multicentre study carried out in Italy showed that five years after primary immunisation, Hexavac albeit less immunogenic than Infanrix, induced immune memory. ${ }^{44}$ Therefore, it can be inferred that if such vaccinated children are exposed to HBV, the immune memory rapidly induces a vigorous anamnestic response that prevents acute disease and the development of a chronic carrier state. In other words, even children who have lost protective antibody concentrations may still maintain T-cell memory that is able to trigger antiHBs production by B cells following activation by revaccination or by natural exposure to $\mathrm{HBV}^{45,46}$ Thus, the main message from this finding is that routine booster doses of vaccine do not seem necessary to sustain immunity in children primed with hexavalent vaccines. However, additional follow up is needed to see whether immunological memory persists during adolescence and adulthood, when risk of HBV exposure does significantly increase, or whether a booster may be needed later in life to maintain lifelong protection.

\section{Conclusions and what the future holds}

Vaccination has proved successful in Italy. Our findings compare well with data reported in previously highly endemic countries (e.g. Taiwan, the Gambia, Alaska) where the impact of vaccination in terms of reduction in incidence, in carrier rate, and in HBV-related mortality has been highly impressive.

Economic analyses show enormous benefits to the country at large as well as to individuals, and projections up to 2060 indicate that elimination of hepatitis B will have paid for itself and covered the costs of the campaigns many times over.

The Italian vaccination programme must continue until every generation has been immunized. Increasing the $\mathrm{HBV}$ vaccination coverage in high-risk-groups, including households of HBsAg carriers and immigrants coming from highly endemic countries, must be a priority. New antiviral treatments are being introduced, offering hope to the 600,000 or so people (mainly over the fifth decade of life) who remain carriers of HBV in the country (compared with 2 million 20 years ago) and those who still contract the disease. The success of Italy's hepatitis B vaccination programme should pave the way for the introduction of new vaccines both nationally and in other countries, as well as reinvigorating existing campaigns against vaccine-preventable diseases. 
Correspondence: Prof. Alessandro R. Zanetti, Dipartimento di Sanità Pubblica - Microbiologia - Virologia, Università di Milano, via C. Pascal 36, 20133 Milano, Italy.

Tel. +39.02.50315126 - Fax: +39.02 .50315120 .

E-mail: alessandro.zanetti@unimi.it

Key words: HBV, hepatitis B, vaccination, long-term immunity.

Acknowledgments: data reported in this paper were presented at a national Meeting, Towards the elimination of hepatitis B: celebrating 20 years of vaccination in Italy, held in Milano (19 November 2011) under the high patronage of the President of the Italian Republic. Distinguished figures from the world of Italian Public Health as well as international experts participated and paid tribute to the work of those who had contributed to the saving of lives, prevention of suffering, and the savings made to the State.

Conflict of interest: the authors declare no conflicts of interest.

Received for publication: 16 February 2012.

Accepted for publication: 20 February 2012.

(C) Copyright L. Romanò et al., 2012

Licensee PAGEPress, Italy

Journal of Public Health Research 2012; 1:e18

doi:10.4081/jphr.2012.e18

This work is licensed under a Creative Commons Attribution NonCommercial 3.0 License (CC BY-NC 3.0).

\section{References}

1. Recommendation of the Immunization Practices Advisory Committee (ACIP). Inactivated hepatitis B virus vaccine. MMWR Morbid Mortal Wkly Rep 1982;31:317-28.

2. Centers for Disease Control and Prevention. Hepatitis B virus: a comprehensive strategy for eliminating transmission in the United States through universal childhood vaccination: Recommendations of the Immunization Practices Advisory Committee (ACIP). MMWR Rec Rep 1991;40(RR-13):1-19.

3. Expanded Programme on Immunization Global Advisory Group. Wkly Epidemiol Rec 1992;67:11-5.

4. Ascherio A, Zhang S, Hernan M, et al. Hepatitis B vaccination and the risk of multiple sclerosis. N Engl J Med. 2001;344:327-32.

5. Confavreux C, Suissa S, Saddier P, et al. Vaccination and the risk of relapse in multiple sclerosis. N Engl J Med. 2001;344:319-26.

6. Duclos P. Safety of immunization and adverse events following vaccination against hepatitis B. J Hepatol. 2003;39:S83-8.

7. Naismith RT, Cross AH. Does the hepatitis B vaccine cause multiple sclerosis? Neurology 2004;63:772-3.

8. WHO. Global Advisory Committee on Vaccine Safety: Response to the paper by MA Hernan and others in Neurology 14th September 2004 issue entitled "Recombinant hepatitis B vaccine and the risk of multiple sclerosis". Available from: http://www.who.int/ vaccine_safety/topics/hepatitisb/multiple_sclerosis/sep_04/en/

9. Mikaeloff Y, Caridade G, Suissa S, Tardieu M. Hepatitis B vaccine and the risk of CNS inflammatory demyelination in childhood. Neurology 2009;72:873-80.

10. Yuen MF, Lim WL, Chan A0, et al. 18-years follow-up study of a prospective randomized trial of hepatitis $B$ vaccination without booster doses in children. Gastroenterol Hepatol 2004;2:941-5.

11. Chen DS. Long-term protection of hepatitis B vaccine: lessons from Alaska experience after 15 years. Ann Intern Med 2005;142:384-5.

12. McMahon BJ, Bruden DL, Peterson KM, Bulkow LR, Parkinson AJ, Nainan 0, et al. Antibody levels and protection after hepatitis B vaccination: results of a 15-years follow-up. Ann Intern Med 2005;142:333-41.

13. Zanetti AR, Mariano A, Romanò L, et al. Long-term immunogenicity of hepatitis B vaccination and policy for booster: an Italian multicentre study. Lancet 2005;366:1379-84.

14. Lu CY, Ni YH, Chiang BL, et al. Humoral and cellular response to a hepatitis B vaccine booster 15-18 years after neonatal immunization. J Infect Dis 2008;197:1419-26.

15. Alfaleh F, Alshehri S, Alansari S, et al. Long-term protection of hepatitis B vaccine 18 years after vaccination. J Infect 2008;57:404-9.

16. Leuridan E, Van Damme P. Hepatitis B and the need for a booster dose. Clin Infect Dis 2011;53:68-75.

17. van der Sande MA, Waight PA, Mendy M, et al. Long-term protection against HBV chronic carriage of Gambian adolescents vaccinated in infancy and immune response in HBV booster trial in adolescence. PLoS One. 2007;2:e753.

18. Jan CF, Huang KC, Chien YC, greydanus DE, Davies HD, Chiu TY, et al. Determination of immune memory to hepatitis B vaccination through early booster response in college students. Hepatology 2010;51:1547-54.

19. West DJ, Calandra GB. Vaccine induced immunologic memory for hepatitis B surface antigen: implications for policy on booster vaccination. Vaccine 1996;14:1019-27.

20. Banatvala JE, Van Damme P. Hepatitis B vaccine: do we need boosters? J Hepatol 2003;10:1-6.

21. Bauer T, Jilg W. Hepatitis B surface antigen-specific T and B cell memory in individuals who had lost protective antibodies after hepatitis B vaccination. Vaccine 2006;24:572-7.

22. European Consensus Group on Hepatitis B Immunity. Are booster immunisation needed for lifelong hepatitis B immunity? Lancet 2000;355:561-5.

23. Su WJ, Liu CC, Liu DP, et al. Effect of age on the incidence of acute hepatitis B after 25 years of a universal newborn hepatitis B immunization program in Taiwan. J Infect Dis 2012;205:757-62.

24. Zanetti AR, Van Damme P, Shouval D. The global impact of vaccination against hepatitis B: a historical overview. Vaccine 2008;26:6266-73.

25. Ni YH, Huang LM, Chang MH, et al. Two decades of universal hepatitis B vaccination in Taiwan: impact and implication for future strategies. Gastroenterology 2007;132:1287-93.

26. Chang MH, Chen CJ, Lai MS, et al. Universal hepatitis B vaccination in Taiwan and the incidence of hepatocellular carcinoma in children. Taiwan Childhood Hepatoma Study Group. N Engl J Med 1997;336:1855-9.

27. Chang MH, You SL, Chen CJ, et al. Decreased incidence of hepatocellular carcinoma in hepatitis B vaccines: a 20-year follow-up study. J Natl Cancer Inst 2009;101:1348-55.

28. Viviani S, Jack A, Hall AJ, et al. Hepatitis B vaccination in infancy in The Gambia: protection against carriage at 9 years of age. Vaccine 1999;17:2946-50.

29. Ng KP, Saw TL, Baki A, et al. Impact of the expanded program of immunization against hepatitis B infection in school children in Malaysia. Med Microbiol Immunol 2005;194:163-8.

30. McMahon BJ, Dentinger CM, Bruden D, et al. Antibody levels and protection after hepatitis B vaccine: results of a 22-year follow-up study and response to a booster dose. J Infect Dis 2009;200:1390-6.

31. Bergamini F, Zanetti AR, Ferroni P, et al. Immune response to hepatitis $B$ vaccine in staff and patients in renal dialysis units. $J$ Infection 1983;7(Suppl 1):35-40.

32. Piazza M, Da Villa G, Picciotto L, et al. Mass vaccination against hepatitis B in infants in Italy. Lancet 1988;332:1132.

33. Zanetti AR, Tanzi E, Romanò L, Grappasonni I. Vaccination against hepatitis B: the Italian strategy. Vaccine 1993;11:521-4.

34. Stroffolini T, Pasquini P, Mele A, et al. A nationwide vaccination 
programme in Italy against hepatitis B virus infection in infants of hepatitis B surface antigen-carrier mothers. Vaccine 1989;7:152-4.

35. Bonanni P, Bechini A, Tiscione E, et al. Impact of universal vaccination programmes on the epidemiology of hepatitis B: 10 years of experience in Italy. Vaccine 2003;21:685-91.

36. Seieva database. Sistema Informatizzato Malattie Infettive (SIM). Available from: http://www.iss.it/seieva/

37. Da Villa G, Romanò L, Sepe A, et al. Impact of hepatitis B vaccination in high endemic area of south Italy and long-term duration of anti-HBs antibody in two cohort of vaccinated individuals. Vaccine 2007;25:3133-6.

38. Gaeta GB, Stroffolini T, Chiaramonte M, et al. Chronic hepatitis D: a vanishing disease? An Italian multicentre study. Hepatology 2000;32:824-7.

39. Zanetti AR, Tanzi E, Manzillo G, et al. Hepatitis B variant in Europe. Lancet 1988;2:1132-3.

40. Carman WF, Zanetti AR, Karayiannis P, et al. Vaccine-induced escape mutant of hepatitis B virus. Lancet 1990;336:325-9.

41. Hsu HY, Chang MH, Ni YH, et al. No increase in prevalence of hepatitis $B$ surface antigen mutant in a population of children and ado- lescents who were fully covered by universal infant immunization. J Infect Dis 2010;201:1192-200.

42. Francois G, Kew M, Van Damme P, et al. Mutant hepatitis B virus: a matter of academic interest only or a problem with far-reaching implications? Vaccine 2001;19:3799-815.

43. European Medicines Agency. Scientific conclusions and grounds for the suspension of the marketing authorisation of Hexavac presented by the EMEA. Available from: http://www.ema.europa.eu/ docs/en_GB/document_library/EPAR_-_Scientific_Conclusion/ human/000298/WC500074684.pdf

44. Zanetti AR, Romanò L, Giambi C, et al. Hepatitis B immune memory in children primed with hexavalent vaccines and given monovalent booster vaccines: an open-label, randomised, controlled, multicentre study. Lancet Infect Dis 2010;10:755-61.

45. Wenzel J, Jilg W. Loss of antibodies, but not of protection. Lancet Infect Dis 2010;10:738-9.

46. Rosado MM, Scarsella M, Pandolfi E, et al. Switched memory B cells maintain specific memory independently of serum antibodies: the hepatitis B example. Eur J Immunol 2011;41:1800-8. 